\title{
Inconclusive Result
}

National Cancer Institute ( $\mathrm{NCl})$

\section{Source}

National Cancer Institute (NCI). Inconclusive Result.

A negative test result in an individual where a clearly deleterious mutation has not been

found in any family members. The genetic risk status of such an individual must be interpreted in the context of his or her personal and family history. Also called indeterminate result and uninformative result. 